\title{
Direct imaging of glycans in Arabidopsis roots via click labeling of metabolically incorporated azido-monosaccharides
}

\author{
Jorin Hoogenboom ${ }^{1 \dagger}$, Nathalja Berghuis ${ }^{1 \dagger}$, Dario Cramer $^{2}$, Rene Geurts ${ }^{3}$, Han Zuilhof ${ }^{1}$ and Tom Wennekes ${ }^{1,2^{*}}$ (D)
}

\begin{abstract}
Background: Carbohydrates, also called glycans, play a crucial but not fully understood role in plant health and development. The non-template driven formation of glycans makes it impossible to image them in vivo with genetically encoded fluorescent tags and related molecular biology approaches. A solution to this problem is the use of tailor-made glycan analogs that are metabolically incorporated by the plant into its glycans. These metabolically incorporated probes can be visualized, but techniques documented so far use toxic copper-catalyzed labeling. To further expand our knowledge of plant glycobiology by direct imaging of its glycans via this method, there is need for novel click-compatible glycan analogs for plants that can be bioorthogonally labelled via copper-free techniques.
\end{abstract}

Results: Arabidopsis seedlings were incubated with azido-containing monosaccharide analogs of $\mathrm{N}$-acetylglucosamine, $\mathrm{N}$-acetylgalactosamine, L-fucose, and L-arabinofuranose. These azido-monosaccharides were metabolically incorporated in plant cell wall glycans of Arabidopsis seedlings. Control experiments indicated active metabolic incorporation of the azido-monosaccharide analogs into glycans rather than through non-specific absorption of the glycan analogs onto the plant cell wall. Successful copper-free labeling reactions were performed, namely an inverse-electron demand Diels-Alder cycloaddition reaction using an incorporated $\mathrm{N}$-acetylglucosamine analog, and a strain-promoted azide-alkyne click reaction. All evaluated azido-monosaccharide analogs were observed to be non-toxic at the used concentrations under normal growth conditions.

Conclusions: Our results for the metabolic incorporation and fluorescent labeling of these azido-monosaccharide analogs expand the possibilities for studying plant glycans by direct imaging. Overall we successfully evaluated five azido-monosaccharide analogs for their ability to be metabolically incorporated in Arabidopsis roots and their imaging after fluorescent labeling. This expands the molecular toolbox for direct glycan imaging in plants, from three to eight glycan analogs, which enables more extensive future studies of spatiotemporal glycan dynamics in a wide variety of plant tissues and species. We also show, for the first time in metabolic labeling and imaging of plant glycans, the potential of two copper-free click chemistry methods that are bio-orthogonal and lead to more uniform labeling. These improved labeling methods can be generalized and extended to already existing and future click chemistry-enabled monosaccharide analogs in Arabidopsis.

Keywords: Click chemistry, Arabidopsis thaliana, Cell wall, Glycans, L-Arabinofuranose, D-Glucosamine, D-Galactosamine, L-Fucose, Metabolic oligosaccharide engineering

\footnotetext{
*Correspondence: t.wennekes@uu.nl

${ }^{\dagger}$ Equal contributors

${ }^{1}$ Laboratory of Organic Chemistry, Wageningen University, Stippeneng 4, 6708 WE Wageningen, The Netherlands

${ }^{2}$ Department of Chemical Biology and Drug Discovery, Utrecht Institute for

Pharmaceutical Sciences and Bijvoet Center for Biomolecular Research,

Utrecht University, Utrecht, The Netherlands

Full list of author information is available at the end of the article
} 


\section{Background}

All plant cells are covered by a dense layer of carbohydrates (glycans), called the glycocalyx. It is the glycocalyx that is first encountered by other cells, including microbes. Glycans are also found on more than $50 \%$ of plant proteins as an important post-translational modification that directly influences protein functioning [1]. Hence it is not surprising that glycans play essential roles in a myriad of biological processes in all stages of plant development, such as cell-cell communication [2], control of metabolism, growth, stress response [3] and external signalling, thereby also tied to the rhizosphere [4-6]. Glycans thus play a crucial but not well understood role in plant health and disease. Developing techniques to better study plant glycans and increase our understanding of and control over their role is an essential next step in plant sciences.

Due to the non-template driven formation of glycans it is not possible to use genetically encoded proteinbased fluorescent tags to image and study glycans directly. Externally added protein-based probes, usually fluorescently-labeled lectins, image the glycans indirectly and are only able to image glycans exposed on the most outer layer of the cell surface glycocalyx [7, 8].

Another approach, however, exists that allows the direct imaging of plant glycans. Glycans, especially in plants, usually have highly complex and diverse structures containing monosaccharides such as glucose, $N$-acetylglucosamine, galactose, L-arabinose, xylose, L-fucose and 3-deoxy-D-manno-oct-2-ulosonic acid (KDO) [9-11]. Besides their de novo biosynthesis, these monosaccharides and their derivatives can also be recycled by plant cells
[12]. Through uptake of extracellular monosaccharides and by intracellular catabolism of complex plant glycans, these monosaccharides can be recycled through the glycan salvage pathways. Using this recycling pathway the monosaccharides again end up in plant cell-surface glycans and its glycoproteins [13]. Glycans and their conjugates are biosynthesized by glycosyltransferases present in the Golgi apparatus and endoplasmic reticulum (ER). The composition and levels of glycans in the glycocalyx and in proteins depends on the presence and levels of these enzymes and their activated monosaccharide donor substrates [12].

The metabolic incorporation of monosaccharide analogs with a latent imaging tag via these pathways would allow for the direct imaging of plant glycans (Fig. 1) [12]. These incorporated monosaccharide analogs can be visualized and studied through a tag that enables click chemistry, which allows for rapid, specific and versatile covalent labeling of plant glycans with a fluorescent reporter molecule $[14,15]$. This technique is called Metabolic Oligosaccharide Engineering (MOE) and it has already been widely applied for studying glycobiology in various organisms, with the notable exception of plants [16]. Indeed, only in 2012, the first application of MOE with click-compatible monosaccharide analogs in plants was reported by Anderson et al. in which fucosylated plant glycans were fluorescently imaged in Arabidopsis thaliana (Col-0) seedlings [17]. Two other clickcompatible monosaccharide analogs were reported recently, namely, 6-deoxy-alkynyl-glucose that incorporates in Arabidopsis root hair tips [18], and 8-azido-8deoxy-KDO, a probe analogous to KDO that is present in the cell wall pectic polysaccharide, rhamnogalacturonan II

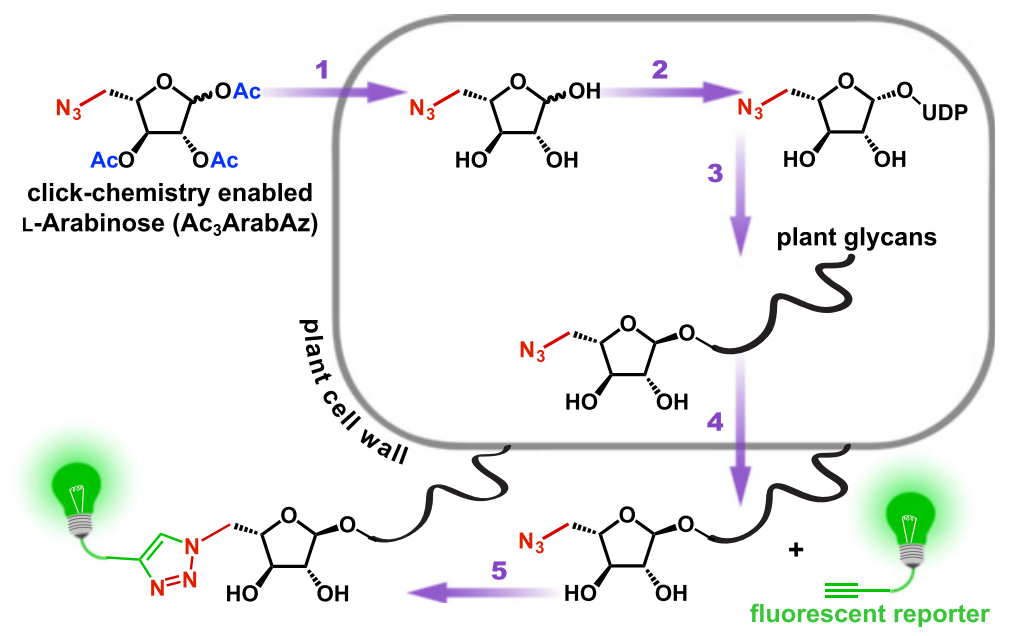

Fig. 1 Metabolic labeling of Arabidopsis cell wall-glycans with azido-monosaccharides. Arabidopsis is grown on MS containing an azido-monosaccharide such as $\mathrm{Ac}_{3} \mathrm{ArabAz}$, which is taken up through the cell wall followed by hydrolysis of the acetyl (Ac) groups by intracellular esterases (1). The resulting ArabAz enters the glycan salvage pathway and is converted to an azido-nucleotide sugar donor (2) that allows its incorporation by glycosyltransferases into plant glycans (3) that end up in plant cell-surface glycans and its glycoproteins (4). Finally, the incorporated glycan can be imaged after a click-reaction with a fluorescent reporter group (5) (see Additional file 14 for high resolution) 
[19]. To further expand our knowledge of plant glycobiology by direct imaging of glycans, there is need for click chemistry-compatible glycan analogs for other plant monosaccharides. In addition, the click chemistry compatible glycan analogs in plants documented so far were labeled using toxic copper-labeling, and future applications would benefit from bio-orthogonal copper-free labeling techniques.

We investigated five glycans: $\mathrm{N}$-acetyl-D-glucosamine, L-fucose and L-arabinose - which are all known to be present in the glycocalyx of Arabidopsis [11, 20] - and $\mathrm{N}$-acetyl-D-galactosamine (GalNAc) and $\mathrm{N}$-acetyl-Dmannosamine. While the latter two glycans are not known to be present in plant glycans, it was recently discovered that UDP-GalNAc is present and transported in the ER of Arabidopsis [21], indicating that GalNAc is metabolized by plants. In this technical advance paper we expand the monosaccharide analog toolbox (Fig. 2) for metabolic labeling of glycans in Arabidopsis seedlings. Furthermore, the glycan analogs reported so far in plants use a $\mathrm{Cu}(\mathrm{I})$-catalyzed cycloaddition, however, this is cytotoxic for Arabidopsis [22] and microbes in soils [23] making this method less suitable for long-term and more complex experiments with living plants. Therefore we investigated the possibilities of bio-orthogonal copper-free click reactions in Arabidopsis roots.

\section{Results and discussion}

\section{Azido-monosaccharides are not toxic at experimental concentrations}

To determine if Arabidopsis seedlings behave differently under normal growth conditions when incubated with our non-natural azido-containing monosaccharide analogs (Fig. 2), their toxicity was evaluated. Earlier reports of metabolic labeling of plant seedlings with monosaccharide analogs have evaluated toxicity by measuring the root length of 8-day old seedlings on MS plates [22, 24]. This toxicity evaluation exposes plant seedlings for several days to high levels of azido-monosaccharides, while the metabolic incorporation experiments are carried out at similar or lower concentrations in a fraction of that time (typically 4-24 h). Accordingly, seedlings were exposed for 8 days to the azido-monosaccharides at concentrations that were used in the different metabolic incorporation experiments (10, 25 and $100 \mu \mathrm{M})$. When compared to seedlings grown on agarose plates with only MS medium, no significant difference was observed (Additional file 1). This shows that azidomonosaccharides do not significantly influence the growth and metabolic processes in Arabidopsis.

\section{$\mathrm{Ac}_{4} \mathrm{GlcNAz}, \mathrm{Ac}_{3} \mathrm{ArabAz}$ and $\mathrm{Ac}_{4} \mathrm{FucAz}$ are incorporated in root cell walls of differentiating Arabidopsis}

$\mathrm{N}$-acetylglucosamine is commonly present in $\mathrm{N}$-glycans of plant cell walls $[11,20]$ and is important for $N$-glycan formation, since it is the first monosaccharide attached to glycoproteins [25]. Therefore metabolic click-mediated labeling of Arabidopsis cell walls was investigated with a $\mathrm{N}$-acetyl-glucosamine analog containing a clickable azide (Ac $\mathrm{Al}_{4}$ GlcNAz; Fig. 2). Ac $\mathrm{C}_{4} \mathrm{GlcNAz}$ was synthesized according to a procedure of Bertozzi and co-workers [26]. $\mathrm{Ac}_{4}$ GlcNAz was dissolved in a $\frac{1}{2} \mathrm{MS}$ medium and used to incubate four day-old Arabidopsis (Col-0) seedlings. The seedlings were incubated with $10,25,50$ or $100 \mu \mathrm{M}$ $\mathrm{Ac}_{4} \mathrm{GlcNAz}$ and control seedlings were incubated with $0.01 \%$ DMSO. After $24 \mathrm{~h}$, seedlings were washed and then transferred for $45 \mathrm{~min}$ to a solution containing Alexa Fluor ${ }^{\circ}$ 488-alkyne and a Click-iT kit solution for the copper-catalyzed labelling. After the labeling and the subsequent washing steps, fluorescence intensity of cell walls was monitored by confocal microscopy. Seedlings incubated with $25 \mu \mathrm{M} \mathrm{Ac} \mathrm{Ac}_{4} \mathrm{GlcNAz}$ showed optimal labeling (Fig. 3a \& Additional file 2). Increased labeling could be reached at higher concentrations, but was not required (Additional file 2B). Control seedlings (Fig. 3e) treated with $0.01 \%$ DMSO did not show auto-fluorescence background signals under these conditions and therefore

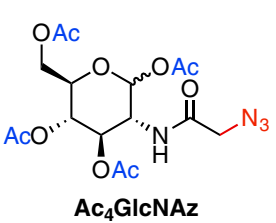<smiles>CC(=O)OCC1OC(OC(C)=O)C(NC(=O)CN)C(OC(C)=O)C1OC(C)=O</smiles><smiles>CC(=O)OCC1O[C@H](OC(C)=O)C(OC(C)=O)[C@H](NC(=O)CN)[C@H]1OC(C)=O</smiles><smiles>CC(=O)O[C@H]1O[C@H](CN)[C@@H](OC(C)=O)[C@H]1OC(C)=O</smiles><smiles>CC(=O)O[C@H]1[C@H](OC(C)=O)[C@@H](CN)O[C@H](OC(C)=O)[C@@H]1OC(C)=O</smiles>

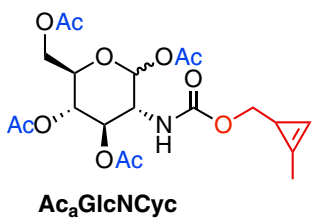

Fig. 2 Chemical structure of click chemistry-enabled monosaccharide analogs that were used in this study (see Additional file 14 for high resolution) 

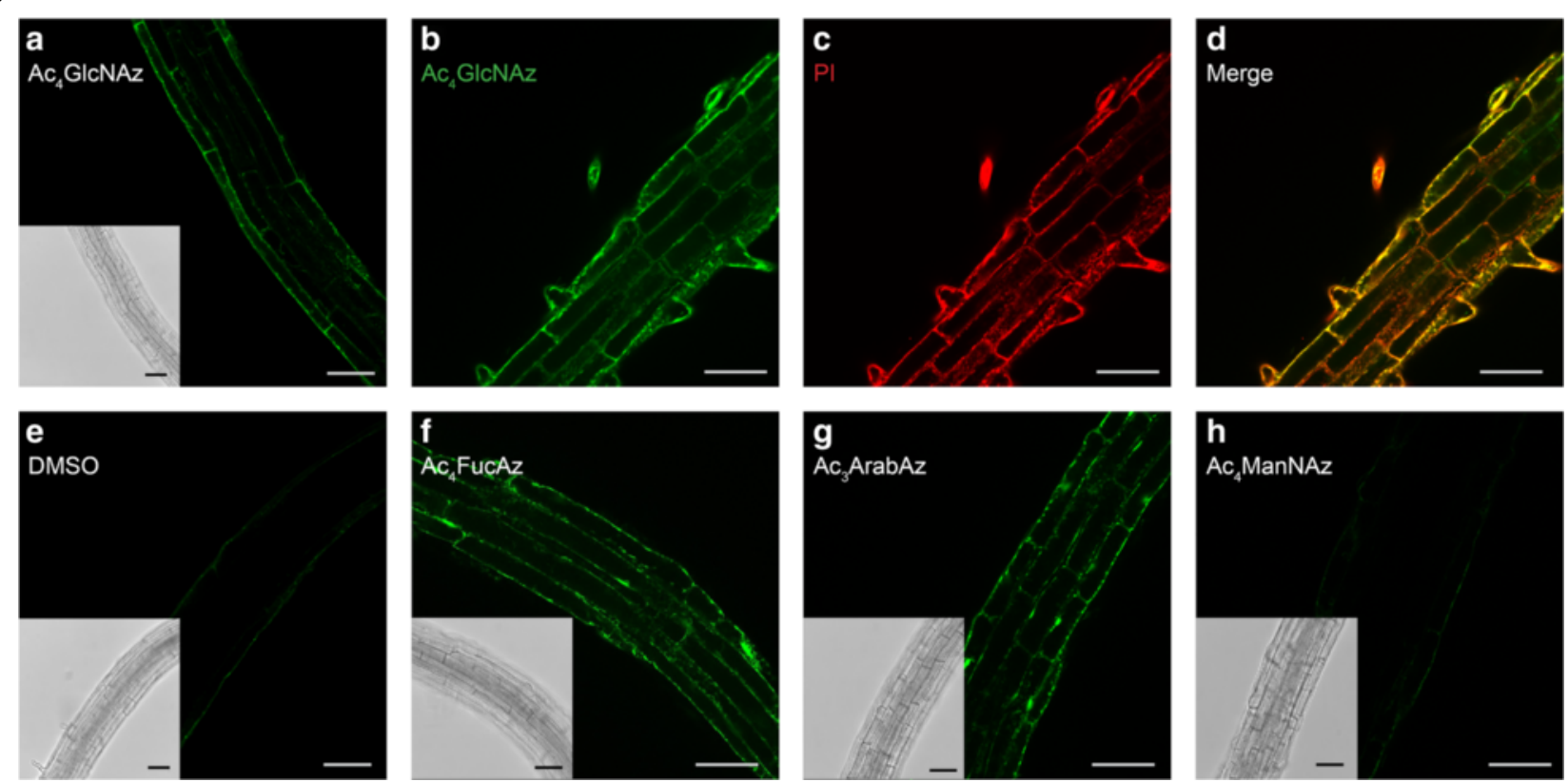

Fig. 3 Optical sections of 4 day old Arabidopsis seedling roots incubated for $24 \mathrm{~h}$ with azido-monosaccharides. Seedlings were incubated with $25 \mu \mathrm{M} \mathrm{Ac}{ }_{4} \mathrm{GlcNAz}(\mathbf{a})$, followed by labeling through a copper-catalyzed click reaction with Alexa Fluor ${ }^{\otimes} 488$ alkyne. Seedling roots treated with Alexa Fluor 488 alkyne-labeled $\mathrm{Ac}_{4} \mathrm{GlcNAz}(25 \mu \mathrm{M}, 24 \mathrm{~h})(\mathbf{b}$, d) were counterstained; Propidium lodide ( $\mathrm{Pl}, 0.05 \%)$ to visualize cell walls (c, d). Yellow color indicates overlap of the two dyes (d). Scale bars $=50 \mu \mathrm{m}$. As a control, seedlings were treated with $0.01 \%$ DMSO (e). Alternatively,

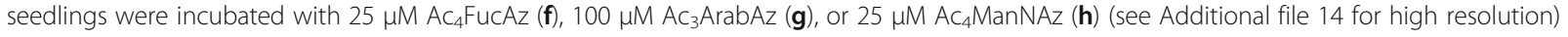

$25 \mu \mathrm{M}$ was used for further labeling experiments with $\mathrm{Ac}_{4}$ GlcNAz.

To determine the subcellular localization of $\mathrm{Ac}_{4}$ Glc$\mathrm{NAz}, \mathrm{Ac}_{4}$ GlcNAz-labeled roots (Fig. 3b) were counterstained with propidium iodide (Fig. 3c) (PI). This revealed that both signals showed overlap (Fig. 3d), indicating a location of $\mathrm{Ac}_{4} \mathrm{GlcNAz}$ in or at the cell walls. During these labeling experiments we focused on studying the transition zone because strong labeling was observed in this region. Directly above this region a decline in labeling was observed, while the meristem zone showed only a slight decrease in labeling.

Encouraged by these results we decided to investigate incorporation and visualization of sugar analogues of Larabinose and L-fucose. Both of these monosaccharides are commonly found in oligosaccharides of plants. More specifically, L-arabinose - mainly present in Arabidopsis as L-arabinofuranose - is one of the most common $\mathrm{O}$ glycan sugars and an important constituent of plant cell wall polysaccharides [27-30]. Furthermore, an alkynylated fucose analog was the first successful metabolically incorporated sugar in Arabidopsis cell walls [22]. Hence, we investigated whether the azido-analogues of L-fucose and L-arabinose may be metabolically incorporated into glycans by Arabidopsis seedling roots. To that end, the two corresponding azido analogues of these sugars were synthesized; $\mathrm{Ac}_{3} \mathrm{ArabAz}$ and $\mathrm{Ac}_{4} \mathrm{FucAz}$ (Fig. 2). $\mathrm{Ac}_{4} \mathrm{Fu}-$ cAz was prepared by acetylating commercially available
6-azido-L-fucose, while the novel $\mathrm{Ac}_{3} \mathrm{ArabAz}$ was prepared according to an adapted procedure of 5-azido-Darabinose by Smellie and co-workers [31]. With both azido-monosaccharides in hand, the feasibility of the incorporations of these compounds was investigated. Similar to the investigation of $\mathrm{Ac}_{4} \mathrm{GlcNAz}$ the optimal incorporation was determined by using different concentrations of $\mathrm{Ac}_{4} \mathrm{FucAz}$ (Additional file 3) and $\mathrm{Ac}_{3} \mathrm{ArabAz}$ (Additional file 4). Clear incorporation of $\mathrm{Ac}_{4} \mathrm{FucAz}$ was observed at the $25 \mu \mathrm{M}$ range (Fig. 3f), whereas reliable incorporation of $\mathrm{Ac}_{3} \mathrm{ArabAz}$ was only observed after incubation at a concentration of $100 \mu \mathrm{M}$ (Fig. 3g). This is most likely due to the relatively high abundance of naturally occurring L-arabinose compared to $\mathrm{N}$-acetylglucosamine and L-fucose. As such, the relatively high concentration of L-arabinose would compete during incorporation of $\mathrm{Ac}_{3} \mathrm{ArabAz}$ at low concentrations of this probe.

L-Arabinofuranosyl residues are incorporated into plant arabinogalactan from UDP-Araf by glycosyltransferases. This nucleotide sugar donor is believed to be biosynthesized exclusively from the thermodynamically more stable pyranosyl form of the same donor; UDPArap. However, ArabAz is not able to convert to its pyranose configuration meaning that the corresponding pyranosyl UDP-ArabAz cannot exist. This raises the question how ArabAz is incorporated. Fincher and coworkers recently reported on the catalytic properties of 
an UDP-arabinose mutase (UAM) enzyme in Barley that catalyzes the multistep reversible UDP-Ara $\rightarrow$ UDP-Araf reaction [32]. They state that a key step in this reaction presumably includes cleavage of the arabinosyl residue from UDP-Ara $p$, which allows opening of the pyranosyl-ring, formation of the furanose ring, and reconnection of the arabinofuranosyl residue to the UDP molecule. Similar UDPmutase enzymes (RGP) have been reported in other plant species, including Arabidopsis [33]. Consequently, ArabAz may be recognized by these enzymes and converted to UDP-ArabAz and thus enable metabolic incorporation.

Next, the results obtained with the three azidomonosaccharides were compared with an azido analog of $\mathrm{N}$-acetyl-D-mannosamine; $\mathrm{Ac}_{4} \mathrm{ManNAz}$ (Fig. $3 \mathrm{~h}$ and Additional file 5). $\mathrm{Ac}_{4} \mathrm{ManNAz}$ differs in only one chiral centre compared to $\mathrm{Ac}_{4} \mathrm{GlcNAz}$, however, no evidence exists in literature that the corresponding monosaccharide (ManNAc) is incorporated in Arabidopsis glycans. In addition, no evidence exist that mannosamine can be used as a precursor for the biosynthesis of other sugar derivatives in plants [34]. Indeed, Arabidopsis seedlings incubated with $\mathrm{Ac}_{4} \mathrm{ManNAz}$ showed no labeling. This confirms that $\mathrm{Ac}_{4} \mathrm{ManNAz}$ is indeed not present in Arabidopsis cell walls and supports the experiments described above that indicated that $\mathrm{Ac}_{4} \mathrm{GlcNAz}, \mathrm{Ac}_{4} \mathrm{FucAz}$ and $\mathrm{Ac}_{3} \mathrm{ArabAz}$ incorporation is mediated by active metabolism.

\section{Azido-monosaccharide incorporation is time-dependent and mediated by passive or active transport}

To investigate if active cellular metabolism is necessary for incorporation of azido-monosaccharides, whole seedlings were killed and fixated by $4 \%$ paraformaldehyde. These fixated seedlings could then be used to distinguish between two scenarios, one where incorporation takes place via an active glycan salvage pathway [12], or alternatively, a scenario where azido-monosaccharides are passively absorbed onto external cell walls. Fixation resulted in slightly more background fluorescence, but the intensity of fixated seedlings incubated with $\mathrm{Ac}_{4} \mathrm{GlcNAz}$ was equal to fixated DMSO control seedlings (Additional file 6). This suggests that $\mathrm{Ac}_{4} \mathrm{GlcNAz}$ is actively incorporated through the plant cell metabolism rather than through non-specific absorption of the azido-monosaccharide to the plant cell wall. Similar results have been reported for alkyne-monosaccharides and a different azido-monosaccharide in Arabidopsis [18, 19, 22].

Next, the optimal incubation time of Arabidopsis seedlings in MS with $25 \mu \mathrm{M} \mathrm{Ac} \mathrm{Ac}_{4} \mathrm{GlcNAz}$ was determined (Fig. 4 \& Additional file 7). Visible incorporation (Fig. 4b) was observed after $4 \mathrm{~h}$ of incubation with $25 \mu \mathrm{M}$ $\mathrm{Ac}_{4} \mathrm{GlcNAz}$, while no incorporation was observed after $2 \mathrm{~h}$ (Fig. 4a). The brightest fluorescence was observed after 6 and $8 \mathrm{~h}$ of incubation (Fig. 4c-d \& Additional file 7). This supports the idea that an active glycan salvage pathway is required for incorporation of $\mathrm{Ac}_{4} \mathrm{GlcNAz}$. In a scenario involving passive adsorption weak fluorescence would already be expected after $2 \mathrm{~h}$ of incubation. Fluorescence decreased after $24 \mathrm{~h}$ incubation, which is most likely due to spreading of $\mathrm{Ac}_{4} \mathrm{GlcNAz}$ through the whole Arabidopsis root or an increased competition with natural $N$-acetyl-D-glucosamine synthesized by the plant itself. The time-dependent incorporation was also investigated for $\mathrm{Ac}_{4} \mathrm{FucAz}$ and $\mathrm{Ac}_{3} \mathrm{ArabAz}$. In contrast to $\mathrm{Ac}_{4} \mathrm{GlcNAz}$, incorporation of $\mathrm{Ac}_{4} \mathrm{FucAz}_{\mathrm{z}}$ and $\mathrm{Ac}_{3} \mathrm{ArabAz}$ was visible after $2 \mathrm{~h}$, but the best incorporation was observed after $24 \mathrm{~h}$ (Additional files 8 and 9).

It is generally believed that the more hydrophobic acetylated monosaccharide probes, compared to their more polar non-acetylated version, end up inside plant cells via passive uptake [17-19]. Roberts et al. reported that root tissues of higher plants rapidly take up D-Glucosamine from aqueous medium for incorporation into root tissue [35]. They also observed active uptake of $\mathrm{N}$-acetyl-D-glucosamine, albeit 10 times slower, via the same pathway [35]. Since this indicated that $N$-acetylglucosamine - not acetylated at any of the hydroxyl groups - is actively taken up by the roots of Arabidopsis, we wondered whether the corresponding non-acetylated GlcNAz could also be incorporated similar to the fully acetylated analog, $\mathrm{Ac}_{4} \mathrm{Glc}-$ NAz. To investigate this, Arabidopsis seedlings were incubated for $24 \mathrm{~h}$ with either $25 \mu \mathrm{M} \mathrm{Ac} \mathrm{Al}_{4} \mathrm{GlNAz}$ or $25 \mu \mathrm{M} \mathrm{GlcNAz}$ (Fig. 5a and b). Incorporation was visible for both $\mathrm{Ac}_{4} \mathrm{GlcNAz}$ and GlcNAz with an almost similar fluorescent strength. This can indicate a maximum uptake for both sugar analogues after $24 \mathrm{~h}$. It also suggests

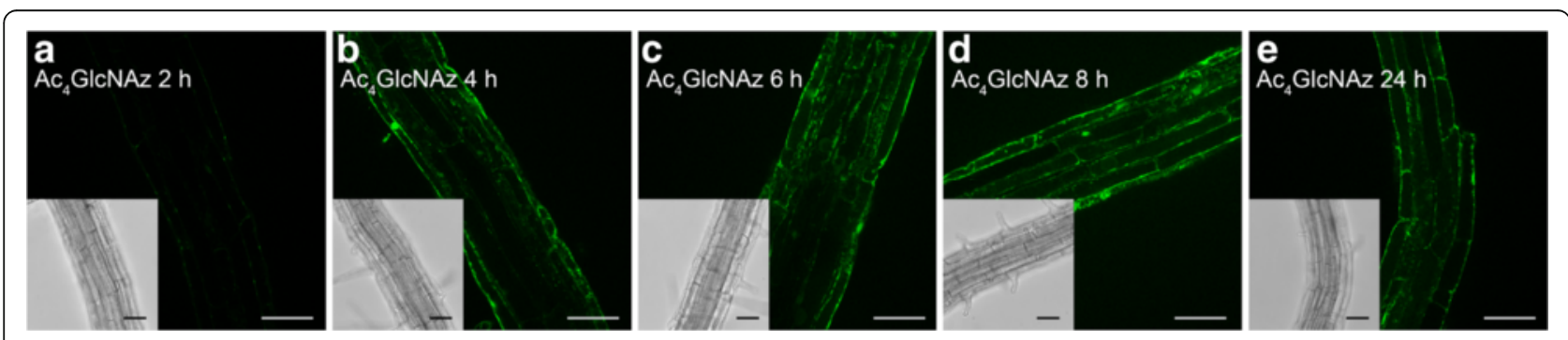

Fig. 4 Optical sections of 4 day old Arabidopsis seedling roots incubated for 2 (a), 4 (b), 6 (c), 8 (d) and 24 h (e) with $25 \mu \mathrm{M} A c_{4} G l c N A z$, followed by labeling through a copper-catalyzed click reaction with Alexa Fluor 488 alkyne. Scale bars $=50 \mu \mathrm{m}$ (see Additional file 14 for high resolution) 


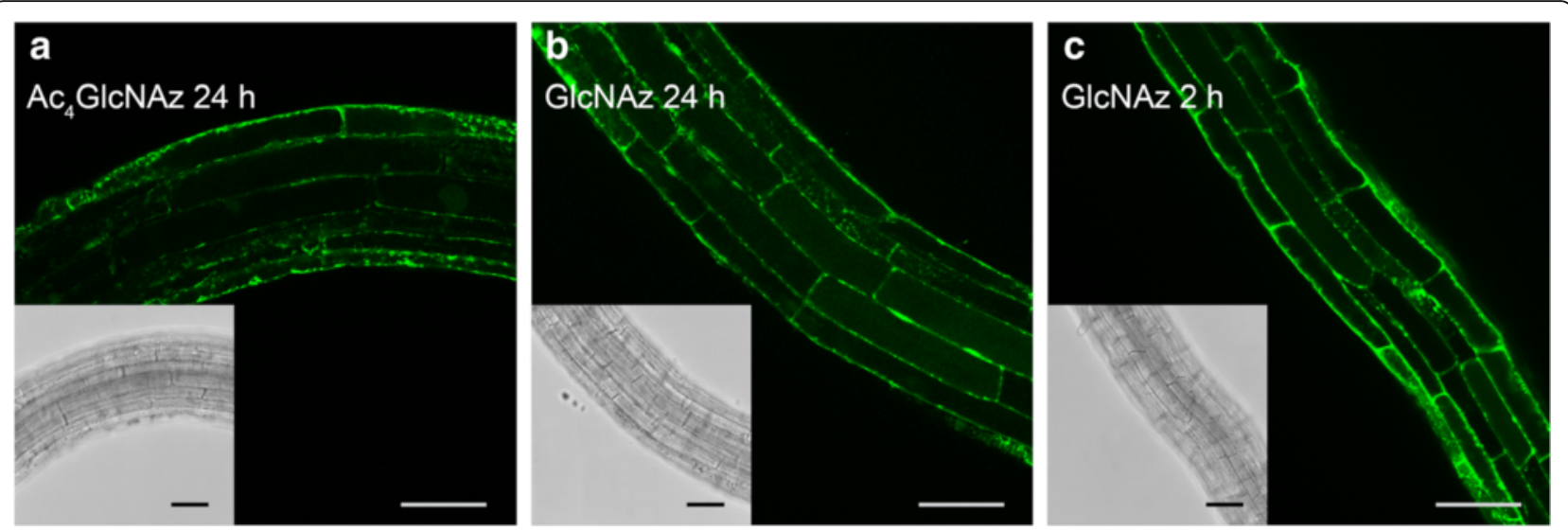

Fig. 5 Optical sections of 4 day old Arabidopsis seedling roots incubated for 24 h with $25 \mu \mathrm{M}$ acetylated Ac 4 GlcNAz (a) or non-acetylated GlcNAz (b), followed by labeling through a copper-catalyzed click reaction with Alexa Fluor ${ }^{\circledast} 488$ alkyne. Early incorporation with GlcNAz was observed with seedlings incubated for $2 \mathrm{~h}$ with $25 \mu \mathrm{M}$ GlcNAz (c). Scale bars $=50 \mu \mathrm{m}$ (see Additional file 14 for high resolution)

that GlcNAz is actively taken up via a cell membrane transport system as its polarity makes passing the fatty non-polar cell membranes via passive transport implausible. Non-acetylated GlcNAz uptake and incorporation was already visible after $2 \mathrm{~h}$ (Fig. 5c), this is in line with previous observations [35], although GlcNAz is not directly comparable with $N$-acetyl-D-glucosamine. To determine the location of $\mathrm{Ac}_{4} \mathrm{GlcNAz}$ and GlcNAz incorporation the seedlings were stained with propidium iodide (PI) after the copper-catalyzed click reaction. Overlap of both incorporated monosaccharides with PI was observed, indicating cell wall labeling (Additional file 10), and no differences in labeling pattern between $\mathrm{Ac}_{4}$ GlcNAz and GlcNAz were observed. Taken together, this indicates that Arabidopsis is capable of active uptake of GlcNAz and it is probably salvaged and incorporated via the same pathway as $\mathrm{Ac}_{4} \mathrm{GlcNAz}$.

\section{Incorporation of $\mathrm{Ac}_{4} \mathrm{GalNAz}$ indicates GalNAc is metabolised in Arabidopsis}

$N$-Acetylgalactosamine (GalNAc) is not documented to be present in Arabidopsis glycans [12], while GalNAc is found in several other higher plants $[36,37]$ and in $N$ - glycans of algae [38]. Glycosylation with GalNAc in Arabidopsis has only been documented in genetically engineered plant cell systems of this plant species [39]. Still, while it is not known whether GalNAc is incorporated into glycans, there is evidence for a UDP-GlcNAc nucleotidyltransferase in Arabidopsis that is capable of converting GalNAc-1-P into its corresponding UDP-GalNAc [40]. In addition, a transporter was recently discovered in Arabidopsis that is capable of transporting both UDP-GlcNAc and UDP-GalNAc [21]. The presence of both an UDP-GalNAc transporter and the GalNAc-compatible transferase indicates that GalNAc might be salvaged or metabolized by Arabidopsis. For this reason, we investigated if this glycan metabolism could potentially be studied with a GalNAc-derived azido-monosaccharide, $N$-azidoacetyl-galactosamine ( $\left.\mathrm{Ac}_{4} \mathrm{GalNAz}\right) . \mathrm{Ac}_{4} \mathrm{GalNAz}$ was prepared according to a procedure described by Bertozzi et al. [26] and then co-incubated with Arabidopsis seedlings for $24 \mathrm{~h}$ using different concentrations (2.5-100 $\mu \mathrm{M}$; Additional file 11). An incorporation signal for $\mathrm{Ac}_{4}$ GalNAz was observed after $24 \mathrm{~h}$ (Additional file 11). However, the incubation time was prolonged because we observed lower fluorescence compared to the other azido-monosaccharides that we studied using the

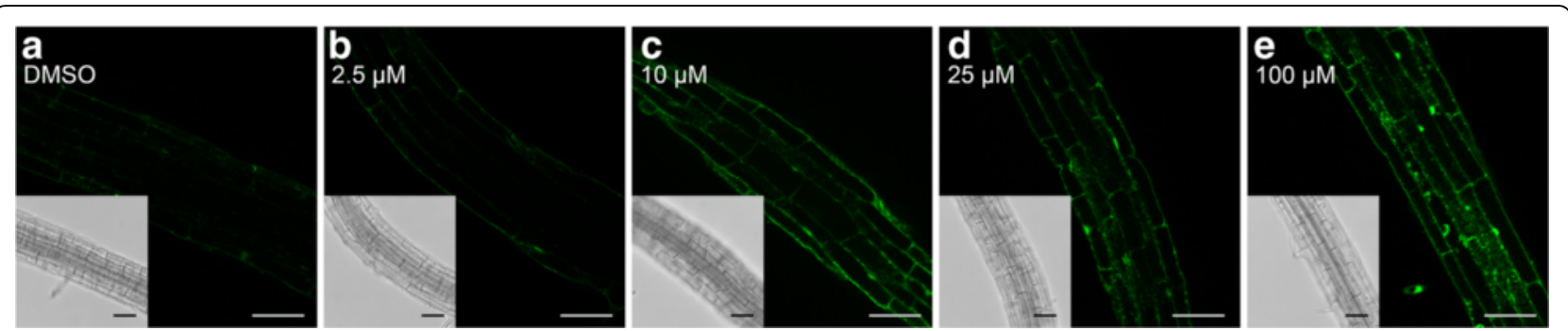

Fig. 6 Optical sections of 4 day old Arabidopsis seedling roots incubated for 48 h with $2.5 \mu \mathrm{M}$ (b), $10 \mu \mathrm{M}$ (c), $25 \mu \mathrm{M}$ (d), and $100 \mu \mathrm{M}$ (e) Ac4GalNAz, followed by labeling through a copper-catalyzed click reaction with Alexa Fluor ${ }^{\circledR} 488$ alkyne. As a control, seedlings were treated with $0.01 \%$ DMSO (a). Scale bars $=50 \mu \mathrm{m}$ (see Additional file 14 for high resolution) 
same incubation time. Increasing the incubation time to $48 \mathrm{~h}$ indeed improved the incorporation (Fig. 6).

This might indicate that salvage and incorporation of $\mathrm{Ac}_{4} \mathrm{GalNAz}$ - compared to the monosaccharide analogs known to be present in Arabidopsis glycans - takes place via a lengthier pathway. The pathway may include an unknown epimerase that converts $\mathrm{N}$-acetylgalactosamine, or a derivative thereof, to the corresponding $\mathrm{N}$-acetylglucosamine epimer. An epimerase has been discovered in barley that reversibly interconverts UDP-GalNAc and UDPGlcNAc and of which a homolog exist in Arabidopsis [41]. Maximum incorporation with $25 \mu \mathrm{M} \mathrm{Ac} \mathrm{AalNAz}_{4}$ was observed in a time-frame of $24 \mathrm{~h}$ (Additional file 11d), whereas $100 \mu \mathrm{M} \mathrm{Ac} \mathrm{Ac}_{4} \mathrm{GalNAz}$ was required (Fig. 6e) to reach saturation with an incubation time of $48 \mathrm{~h}$. The relative high concentration required after $48 \mathrm{~h}$, is consistent with the other lengthier time incubation experiments with azido-monosaccharides.

\section{Copper-free click reactions are good alternatives to label glycans in Arabidopsis roots}

A drawback of the studies reported until now that use monosaccharide probes to image plant glycans is that they all use a copper-catalyzed click reaction to attach the fluorescent reporter group to the metabolically incorporated glycans. The copper required to catalyze this reaction is known to be toxic to Arabidopsis and therefore might influence the outcome of the labeling and imaging experiments in which it is used. This side effect is indeed also observed by us in the slightly inhomogeneous labeling after the copper-catalyzed click reaction, which damages the cell wall and in a few instances also caused minor internal labeling. The toxic effect that copper has on Arabidopsis seedlings was also observed by Anderson and coworkers $[18,22]$, who applied copper-catalyzed reactions to label alkyne-monosaccharides. To circumvent the use of copper ions, an alternative copper-free click reaction, the so-called strain-promoted alkyne-azide cycloaddition (SPAAC) has been developed, which is bio-orthogonal and can be applied to living cells [42]. It has not been applied towards azidomonosaccharide analog probes in Arabidopsis so far. This reaction is still rapid enough for biological applications, for instance when an azide-containing probe is reacted with an aliphatic cyclooctyne $(\mathrm{BCN})$ [43] or dibenzocyclooctyne (DBCO) [44, 45]. Labeling of azido-monosaccharides via SPAAC has an advantage compared to the copper-catalyzed click reaction, since it does not damage living cells. However, while the alkyne-monosaccharides reported earlier cannot utilize SPAAC, our azido-monosaccharide probes do have the potential to be labeled through this click reaction. To investigate copper-free labeling of plant glycans via SPAAC, seedlings were labeled after incubation with $\mathrm{Ac}_{4} \mathrm{GlcNAz}$ $(25 \mu \mathrm{M}, 24 \mathrm{~h}), \mathrm{Ac}_{4} \mathrm{FucAz}(25 \mu \mathrm{M}, 24 \mathrm{~h}), \mathrm{Ac}_{3} \mathrm{ArabAz}$ $(100 \mu \mathrm{M}, 24 \mathrm{~h})$ or $\mathrm{Ac}_{4} \mathrm{GalNAz}(25 \mu \mathrm{M}, 24 \mathrm{~h})$ with a solution containing $1 \mu \mathrm{M}$ DBCO-PEG4-ATTO-488 in MS for $1 \mathrm{~h}$. The resulting metabolically-labeled seedlings showed bright fluorescence and low background (Fig. 7a-d). In contrast, seedlings incubated with $0.01 \%$ DMSO showed only background fluorescence (Fig. 7e).

Comparison of seedlings labeled with $\mathrm{Ac}_{4} \mathrm{GlcNAz}$ and $\mathrm{Ac}_{3} \mathrm{ArabAz}$ with propidium iodide-labeled seedlings showed excellent overlap, indicating incorporation of the azido-monosaccharides in the cell wall glycans (Fig. $7 \mathrm{~h}-\mathrm{j}$ and Additional file 12). Experiments with a more apolar DBCO-fluorophore without a PEG-spacer and a BCNderived fluorophore were not successful and extensive non-specific absorption of the fluorophore (also as micelles) to the cell wall was observed.

In addition to SPAAC, other bio-orthogonal copper-free click reactions are also known. The inverse electron demand Diels-Alder (invDA) reaction between tetrazines and strained alkenes/alkynes has gained popularity as a very fast and bio-orthogonal complementary reaction to SPAAC [46]. We investigated whether this reaction could also be used for labeling plant glycans. Tetrazines conjugated to a fluorescent reporter group are typically used for labeling and we choose the smallest possible tetrazine reaction partner, a methyl-cyclopropene, as a chemical handle on an $\mathrm{N}$-acetylglucosamine derivative. Known GlcNAc derivative with a methyl-cyclopropene (GlcNCyc), was prepared via an adapted procedure of Prescher [47] and Wittmann and co-workers [48]. Arabidopsis seedlings incubated with GlcNCyc for $24 \mathrm{~h}$ showed bright fluorescence, when clicked with $15 \mu \mathrm{M}$ Tetrazine-ATTO-488 (Fig. 7g), while a DMSO control did not show appreciable fluorescence (Fig. 7f). Besides, compared to the other clickable dyes used in this study, it was observed that Alexa ${ }^{\circ}$ Fluor-tetrazine was less prone to stick to the cell wall and more water soluble than alkyne and $\mathrm{DBCO}$ dyes. This has the advantage that the fluorophore can be used at higher concentrations. These preliminary experiments with the SPAAC and invDA copper-free click reactions resulted in a more uniform staining. In addition, these mild labeling reactions do not require cytotoxic copper, which enables experiments that go beyond snapshot images of plant seedlings.

\section{Conclusions}

In this study, the toolbox of clickable monosaccharide analogs for glycan labeling in Arabidopsis seedlings has been expanded to allow the incorporation and direct visualization of five relevant plant monosaccharide analogs in complex cell wall-bound glycans. The clickable glycan analogs $\mathrm{Ac}_{4} \mathrm{GlcNAz}, \mathrm{Ac}_{3} \mathrm{ArabAz}, \mathrm{Ac}_{4} \mathrm{FucAz}$ and $\mathrm{Ac}_{4} \mathrm{GalNAz}$ were successfully metabolically incorporated and visualized in glycans of Arabidopsis seedling roots. The novel $\mathrm{Ac}_{3} \mathrm{ArabAz}$ for the first time allows for direct imaging of L-arabinose, one of the most common plant $O$-glycans and an important constituent of plant cell wall 

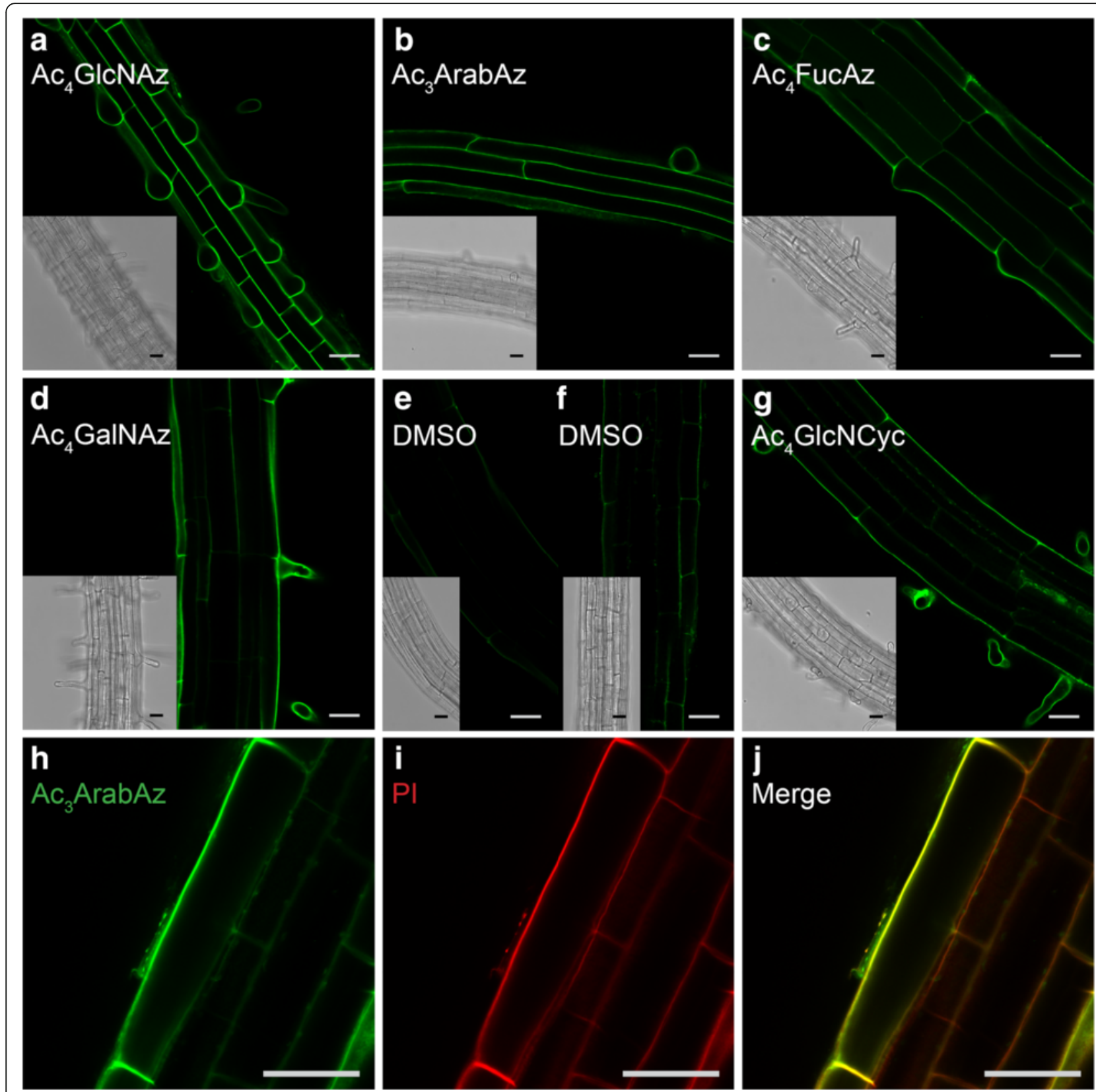

Fig. 7 Optical sections of 4 day old Arabidopsis seedling roots incubated for $24 \mathrm{~h}$ with $25 \mu \mathrm{M}$ acetylated $\mathrm{Ac}_{4} \mathrm{GlcNAz}$ (a), $100 \mu \mathrm{M} \mathrm{Ac} \mathrm{ArabAz}_{3}$ (b), $25 \mu \mathrm{M}$ $\mathrm{AC}_{4} \mathrm{FuCAz}(\mathbf{c}), 25 \mu \mathrm{M} \mathrm{Ac}{ }_{4} \mathrm{GalNAz}(\mathbf{d})$ or $50 \mu \mathrm{M}$ GlcNCyc (g) followed by labeling through strain-promoted alkyne-azide cycloaddition with DBCO-PEG4ATTO-488 (a-d) or an inverse electron demand Diels-Alder click reaction with Tetrazine-ATTO-488 (g). As a control, seedlings were treated with $0.01 \%$ DMSO followed by labeling through strain-promoted alkyne-azide cycloaddition with DBCO-PEG4-ATTO-488 (e) or an inverse electron demand Diels-Alder click reaction with Tetrazine-ATTO-488 (f). Seedling roots treated with DBCO-PEG4-ATTO-488 labeled AC ArabAz (100 $\mu \mathrm{M}, 24 \mathrm{~h})(\mathbf{f}$, j) counterstained Propidium lodide (PI, $0.05 \%$ ) to visualize cell walls (i, j). Yellow color indicates overlap of the two dyes (j). Scale bars $=25 \mu \mathrm{m}$ (see Additional file 14 for high resolution)

polysaccharides [27-30]. The incorporation of $\mathrm{Ac}_{4} \mathrm{Gal}-$ NAz we observe supports the possibility of an epimerase in Arabidopsis that converts GalNAz to GlcNAz. During the preparation of this manuscript Chen and coworkers reported on the metabolic incorporation and imaging of $N$-linked glycans in Arabidopsis with $\mathrm{Ac}_{4}$ GlcNAz [24]. Our here reported results with this azido-monosaccharide are in correspondence with their work, and provide additional details on $\mathrm{Ac}_{4} \mathrm{GlcNAz}$ metabolic incorporation and imaging through the glycan salvage pathway. For example, we show that $\mathrm{Ac}_{4} \mathrm{GlcNAz}$ is already being incorporated after $4 \mathrm{~h}$ and that GlcNAz (non-acetylated) can also be salvaged, probably via active transport, within $2 \mathrm{~h}$. Finally, earlier reports on the metabolic incorporation and imaging 
of monosaccharide analogs, including $\mathrm{Ac}_{4} \mathrm{GlcNAz}$, rely solely on labeling through copper-catalyzed click chemistry. Although copper-catalyzed click reactions often work well, the toxicity of copper here led to damage of the cell wall, emphasizing the need for copper-free clickable analogs for long-term or spatiotemporal experiments. We here show for the first time that the strain-promoted azide-alkyne cycloaddition (SPAAC) and inverse electron demand Diels-Alder (invDA) click reactions allow for improved imaging of metabolic labeling with our azido-monosaccharides and a cyclopropene-GlcNAc derivative. The application of these improved copper free labeling methods can be generalized and extended to already existing and future click chemistry-enabled monosaccharide analogs in Arabidopsis. Taken together with the fact that the SPAAC and invDA reactions are bio-orthogonal and orthogonal with respect to each other, this will allow for in vivo and dual plant glycan labeling applications. Overall our results here and other recently published studies $[18,19,24]$ promise a bright future for the Metabolic Oligosaccharide Engineering (MOE) methodology to enable the direct spatiotemporal imaging of complex glycans in living plants [16].

\section{Methods}

\section{Growth of Arabidopsis Thaliana}

Wild type Arabidopsis thaliana (Col-0) seeds were surface sterilized in a mixture of commercial bleach and ethanol $(\mathrm{v} / \mathrm{v} ; 1 / 4)$ for $15 \mathrm{~min}$ followed by washing with ethanol (2 times) and drying. First a cold shock was applied on all sterilized seeds by placing them in a fridge $\left(5^{\circ} \mathrm{C}\right)$ for at least 2 days with a maximum of one week while on filter paper, pre-wetted with $2 \mathrm{~mL}$ Milli-Q water, in a petri dish. Seeds were grown on half Murashige and Skoog medium (MS) [49] with vitamins in a petri dish (0.8\% plant agar) in a climate room on the shelf lit by Philips $36 \mathrm{~W} / 840$ lamps $\left(120 \mu \mathrm{mol} / \mathrm{m}^{2} \mathrm{~s}\right)$ under long-day conditions $(16 \mathrm{~h} \mathrm{light} / 8 \mathrm{~h}$ dark) at $22{ }^{\circ} \mathrm{C}$. Young seedlings of 4 or 5 days old were used for incubation experiments.

\section{Incubation of Arabidopsis}

Five young seedlings were put together in single well of a 24-well plate containing click-compatible azido-monosaccharide in half MS. After incubation time, 5 wells were filled with $2 \mathrm{~mL}$ half MS medium containing $0.05 \%$ Tween 20. Plants were dipped in each well for $15 \mathrm{~s}$ to wash away the excess of azido-monosaccharide. The seedlings were directly transferred to a new 24-well plate for labeling through either a 1) copper-catalyzed click-labeling 2) a SPAAC labeling or 3) a Diels alder-cycloaddition labeling.

\section{Copper-catalyzed click-labeling}

Click-iT cell reaction kit (supplier: Invitrogen) was used for all copper-catalyzed "click" reactions. The labeling was carried out according to the procedure in the manual of
Invitrogen except for the reaction time that was prolonged to $45 \mathrm{~min}$. For the Alexa-fluor 488 fluorophore a concentration of $0.1 \mu \mathrm{M}$ was found to be the most optimal. The excess of fluorophore was removed by washing the seedlings $4 \times$ in $2 \mathrm{~mL}$ half MS containing $0.05 \%$ Tween 20. Duration of the sequential washings steps were respectfully 5 , 10, 5 and $10 \mathrm{~min}$. After washing the seedlings were stored for with a maximum time of $2 \mathrm{~h}$ in half MS (not containing Tween 20) before visualization by confocal microscopy.

\section{SPAAC labeling}

SPAAC reactions were performed in $2 \mathrm{~mL}$ of $1 \mu \mathrm{M}$ DBCOPEG4-ATTO-488 in half MS medium. Reaction time was $1 \mathrm{~h}$. Washing and storage was similar to the coppercatalyzed click reaction described above. The washing times were prolonged to $4 \times 10 \mathrm{~min}$.

\section{Diels-Alder cycloadditions}

Reactions were performed in $1 \mathrm{~mL}$ of $15 \mu \mathrm{M}$ TetrazineATTO-488 in half MS medium. All other procedures were similar to the SPAAC reactions described above.

\section{Seedling fixation with paraformaldehyde}

As a negative control, seedlings were fixated in $4 \%$ paraformaldehyde solution in PBS (commercially available). Five seedlings were put together in $2 \mathrm{~mL}$ of the paraformaldehyde solution for $30 \mathrm{~min}$. Afterwards seedlings were washed two times in $2 \mathrm{~mL} 0.5 \mathrm{MS}$ before incubation with clickcompatible azido-monosaccharides as discussed before.

\section{Toxicity test}

Toxicity tests were performed based on growth of the plant. Agar plates containing the described azidomonosaccharides analogs were used for the growth experiments with young Arabidopsis seedlings for 8 days. Azido-monosaccharides were added after sterilization of the medium, when it was cooled down to approximately $60{ }^{\circ} \mathrm{C}$ and before pouring the medium in a petri dish. Arabidopsis seedlings were subsequently germinated and grown on agar plates containing the different azidomonosaccharide solutions in $1 / 2$ MS with $0.8 \%$ plant agar. After 8 days of growth, the white part of the root was measured from leaves till root tip.

\section{Microscopy and image analysis}

Roots of seedlings were imaged with a Leica TCS SP8 confocal microscope (488 $\mathrm{nm}$ laser excitation, 534-571 emission filter and 600-650 emission filter for PI) using a 40X water immersion objective. Image J was used to process images. All images within the same experiment were adjusted to the same color balance. Mean fluorescence was calculated in Image J (rsbweb.nih.gov/ij) using freehand tool to select the cell boundary of epidermal cells and to measure the mean pixel intensity. The standard deviation 
was determined based on the difference in the fluorescence intensity throughout the cells of a seedling. Data of those cells were collected from 3-4 seedlings per treatment and imaged using identical exposure settings.

\section{General information and methods for synthesis}

$\mathrm{Ac}_{4} \mathrm{GlcNAz}, \mathrm{Ac}_{4} \mathrm{GalNAz}$ and GlcNCyc were prepared according to literature procedures [26]. GlcNCyc was prepared according to a literature procedure by Presher [47] and Wittmann et al. [48], while the synthesis of one of the intermediates in this synthesis - the cyclopropane tag - has been adapted and described in Additional file 13. The synthesis of $\mathrm{Ac}_{4} \mathrm{FucAz}, \mathrm{Ac}_{3} \mathrm{ArabAz}$ are described in Additional file 13.

\section{Additional files}

\section{Additional file 1: Evaluation of toxicity of azido-monosaccharides.}

(DOCX $22 \mathrm{~kb}$ )

Additional file 2: Concentration-dependent $\mathrm{Ac}_{4} \mathrm{Gl} \mathrm{CNAz}$ incorporation. (DOCX 207 kb)

Additional file 3: Concentration-dependent $\mathrm{Ac}_{4} \mathrm{FuCAz}$ incorporation. (DOCX $338 \mathrm{~kb}$ )

Additional file 4: Concentration-dependent $\mathrm{Ac}_{3} \mathrm{ArabAz}$ incorporation. (DOCX $351 \mathrm{~kb}$ )

Additional file 5: Optical sections of the control experiments of $\mathrm{Ac}_{4}$ ManNAz incorporation. (DOCX $338 \mathrm{~kb}$ )

Additional file 6: Control experiments with paraformaldehyde fixed seedlings. (DOCX $319 \mathrm{~kb}$ )

Additional file 7: Quantified time-dependent GlcNAz incorporation. (DOCX $25 \mathrm{~kb}$ )

Additional file 8: Time-course of $\mathrm{AC}_{4} \mathrm{FuCAz}$ incorporation in elongating root cells. (DOCX $287 \mathrm{~kb})$

Additional file 9: Time-course of $\mathrm{Ac}_{3} \mathrm{Arab} A z$ incorporation in elongating root cells. (DOCX $285 \mathrm{~kb}$ )

Additional file 10: Comparison of non-acetylated $\mathrm{Ac}_{4} \mathrm{Gl} C \mathrm{CN}$ z labeled seedlings with PI stain. (DOCX $775 \mathrm{~kb}$ )

Additional file 11: Concentration-dependent $\mathrm{Ac}_{4} \mathrm{GalNAz}$ incorporation (24 h incubation). (DOCX $322 \mathrm{~kb}$ )

Additional file 12: Comparison of $\mathrm{Ac}_{4} \mathrm{Gl} \mathrm{CNAz}$ and $\mathrm{AC}_{3} \mathrm{ArabAz}$ labeled seedlings with PI stain. (DOCX $1277 \mathrm{~kb})$

Additional file 13: Synthesis procedures and characterization data for azido-monosaccharides. (DOCX $1338 \mathrm{~kb}$ )

Additional file 14: Figures in high resolution. (ZIP $22425 \mathrm{~kb}$ )

\section{Abbreviations}

$\mathrm{Ac}_{3} \mathrm{ArabAz}$ : 1,2,3, Tri-O-acetyl-5-azido-5-deoxy-L-arabinofuranose; Ac $_{4}$ FucAz: 1,2,3,4-tetra-O-acetyl-6-azido-L-fucose; Ac ${ }_{4}$ GalNAz: 1,3,4,4-tetra-Oacetyl-N-azidoacetyl-a, $\beta$-D-galactosamine; $\mathrm{Ac}_{4} \mathrm{Gl}$ CNAz: 1,3,4,4-tetra-O-acetyl-Nazidoacetyl-a, $\beta$-D-glucosamine; Ac ${ }_{4}$ ManNAz: 1,3,4,4-tetra-O-acetyl-N-

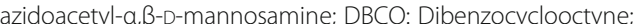
DMSO: Dimethyl sulfoxide; GalNAc: N-acetyl-D-galactosamine; GIcNAz: Nazidoacetylglucosamine; GlcNCyc: 1,3,4,4-tetra-O-acetyl-Nmethylcyclopropene-a, $\beta$-D-glucosamine; PI: Propidium iodide; SPAAC: Strain promoted azido-alkyne click chemistry

\section{Acknowledgements}

The authors thank Martijn Fiers for support during trial experiments and Martinus Schneijderberg, Olga Kulikova for assistance during experiments.

\section{Funding}

This work was financially supported by the Netherlands Foundation for Scientific Research (NWO) via ChemThem: Chemical Biology grant (728.011.105) and VIDI grant (723.014.005).

\section{Authors' contributions}

Conceived and designed the experiments: JH, NB, RG and TW. Performed the experiments and compiled the data: $\mathrm{NB}, \mathrm{JH}$ and DC. Analyzed the data: $\mathrm{JH}$, NB, RG and TW. Wrote the paper: JH, NB, RG, HZ and TW. All authors have read and approved this manuscript.

\section{Competing interests}

The authors declare that they have no competing interests.

\section{Consent for publication}

Not applicable.

Ethics approval and consent to participate Not applicable.

\section{Author details}

${ }^{1}$ Laboratory of Organic Chemistry, Wageningen University, Stippeneng 4, 6708 WE Wageningen, The Netherlands. ²Department of Chemical Biology and Drug Discovery, Utrecht Institute for Pharmaceutical Sciences and Bijvoet Center for Biomolecular Research, Utrecht University, Utrecht, The Netherlands. ${ }^{3}$ Department of Plant Science, Laboratory of Molecular Biology, Wageningen University, Droevendaalsesteeg 1, 6708 PB Wageningen, The Netherlands.

Received: 15 July 2016 Accepted: 26 September 2016

Published online: 10 October 2016

\section{References}

1. Apweiler R, Hermjakob H, Sharon N. On the frequency of protein glycosylation, as deduced from analysis of the SWISS-PROT database. Biochim Biophys Acta. 1999;1473:4-8.

2. Gabius HJ, Andre S, Kaltner H, Siebert HC. The sugar code: functional lectinomics. Biochim Biophys Acta. 2002;1572:165-77.

3. Rolland F, Baena-Gonzalez E, Sheen J. Sugar sensing and signaling in plants: conserved and novel mechanisms. Annu Rev Plant Biol. 2006:57:675-709.

4. Nguema-Ona E, Coimbra S, Vicré-Gibouin M, Mollet J-C, Driouich A. Arabinogalactan proteins in root and pollen-tube cells: distribution and functional aspects. Ann Bot. 2012:110:383-404

5. Bais HP, Weir TL, Perry LG, Gilroy S, Vivanco JM. The role of root exudates in rhizosphere interactions with plants and other organisms. Annu Rev Plant Biol. 2006:57:233-66.

6. Grobelak A, Napora A, Kacprzak M. Using plant growth-promoting rhizobacteria (PGPR) to improve plant growth. Ecol Eng. 2015;84:22-8.

7. Chesson A, Gardner PT, Wood TJ. Cell wall porosity and available surface area of wheat straw and wheat grain fractions. J Sci Food Agric. 1997;75:289-95.

8. Rapoport TA. Protein translocation across the eukaryotic endoplasmic reticulum and bacterial plasma membranes. Nature. 2007:450:663-9.

9. Boundy JA, Wall JS, Turner JE, Woychik JH, Dimler RJ. A mucopolysaccharide containing hydroxyproline from corn pericarp. Isolation and composition. J Biol Chem. 1967;242:2410-5.

10. Kalb AJ. The separation of three L-fucose-binding proteins of Lotus tetragonolobus. Biochim Biophys Acta. 1968;168:532-6.

11. Nguema-Ona E, Vicre-Gibouin M, Gotte M, Plancot B, Lerouge P, Bardor M, Driouich A. Cell wall O-glycoproteins and N-glycoproteins: aspects of biosynthesis and function. Front Plant Sci. 2014:5:499.

12. Bar-Peled M, O'Neill MA. Plant nucleotide sugar formation, interconversion, and salvage by sugar recycling. Annu Rev Plant Biol. 2011:62:127-55.

13. Reiter W-D, Vanzin GF. Molecular genetics of nucleotide sugar interconversion pathways in plants. Plant Mol Biol. 2001;47:95-113.

14. Rostovtsev W, Green LG, Fokin W, Sharpless KB. A Stepwise Huisgen Cycloaddition Process: Copper(I)-Catalyzed Regioselective "Ligation" of Azides and Terminal Alkynes. Angew Chem Int Ed. 2002:41:2596-99.

15. Presolski Sl, Hong V, Cho SH, Finn MG. Tailored ligand acceleration of the Cu-catalyzed azide-alkyne cycloaddition reaction: practical and mechanistic implications. J Am Chem Soc. 2010;132:14570-6. 
16. Sminia, T.J., Zuilhof, H, Wennekes, T. Getting a Grip on Glycans: a CurrentOverview of the Metabolic Oligosaccharide Engineering Toolbox. Carbohydr Res. 2016. Advance online publication. doi: 10.1016/j.carres.2016. 09.007

17. Sawa M, Hsu TL, Itoh T, Sugiyama M, Hanson SR, Vogt PK, Wong CH. Glycoproteomic probes for fluorescent imaging of fucosylated glycans in vivo. Proc Natl Acad Sci U S A. 2006;103:12371-6.

18. McClosky DD, Wang B, Chen G, Anderson CT. The click-compatible sugar 6-deoxy-alkynyl glucose metabolically incorporates into Arabidopsis root hair tips and arrests their growth. Phytochemistry. 2016;123:16-24.

19. Dumont M, Lehner A, Vauzeilles B, Malassis J, Marchant A, Smyth K, Linclau B, Baron A, Mas Pons J, Anderson CT, Schapman D, Galas L, Mollet JC, Lerouge P. Plant cell wall imaging by metabolic click-mediated labelling of rhamnogalacturonan II using azido 3-deoxy-d-manno-oct-2-ulosonic acid. Plant J. 2016:85:437-47.

20. Kajiura H, Tanaka R, Kato K, Hamaguchi Y, Seki T, Fujiyama K. N-Glycan structures of glycoproteins in suspension-cultured Arabidopsis thaliana T87 cells. Plant Biotechnol. 2012;29:489-93.

21. Niemann MCE, Bartrina I, Ashikov A, Weber H, Novak O, Spichal L, Strnad M, Strasser R, Bakker H, Schmulling T, Werner T. Arabidopsis ROCK1 transports UDP-GICNAC/UDP-GalNAC and regulates ER protein quality control and cytokinin activity. Proc Natl Acad Sci U S A. 2015;112:291-96.

22. Anderson CT, Wallace IS, Somerville CR. Metabolic click-labeling with a fucose analog reveals pectin delivery, architecture, and dynamics in Arabidopsis cell walls. Proc Natl Acad Sci U S A. 2012;109:1329-34.

23. Kunito T, Saeki K, Oyaizu H, Matsumoto S. Influences of copper forms on the toxicity to microorganisms in soils. Ecotoxicol Environ Saf. 1999;44:174-81.

24. Zhu Y, Wu J, Chen X. Metabolic Labeling and Imaging of N-Linked Glycans in Arabidopsis Thaliana. Angew Chem Int Ed. 2016;55:9301-05.

25. von Schaewen A, Sturm A, O'Neill J, Chrispeels MJ. Isolation of a Mutant Arabidopsis Plant That Lacks N-Acetyl Glucosaminyl Transferase I and Is Unable to Synthesize Golgi-Modified Complex N-Linked Glycans. Plant Physiol. 1993;102:1109-18.

26. Laughlin ST, Agard NJ, Baskin JM, Carrico IS, Chang PV, Ganguli AS, Hangauer MJ, Lo A, Prescher JA, Bertozzi CR. Metabolic Labeling of Glycans with Azido Sugars for Visualization and Glycoproteomics, in Methods Enzymol. New York: Academic Press. 2006;415:230-50.

27. Burget EG, Verma R, Mølhøj M, Reiter W-D. The Biosynthesis of I-Arabinose in Plants: Molecular Cloning and Characterization of a Golgi-Localized UDP$\mathrm{d}$-Xylose 4-Epimerase Encoded by the MUR4 Gene of Arabidopsis. Plant Cell. 2003;15:523-31.

28. Tan L, Varnai P, Lamport DTA, Yuan CH, Xu JF, Qiu F, Kieliszewski MJ. Plant O-Hydroxyproline Arabinogalactans Are Composed of Repeating Trigalactosy Subunits with Short Bifurcated Side Chains. J Biol Chem. 2010;285:24575-83.

29. Saito F, Suyama A, Oka T, Yoko-o T, Matsuoka K, Jigami Y, Shimma Y. Identification of Novel Peptidyl Serine alpha-Galactosyltransferase Gene Family in Plants. J Biol Chem. 2014;289:20405-20.

30. Carpita NC, Gibeaut DM. Structural models of primary cell walls in flowering plants: consistency of molecular structure with the physical properties of the walls during growth. Plant J. 1993;3:1-30.

31. Smellie IA, Bhakta S, Sim E, Fairbanks AJ. Synthesis of putative chain terminators of mycobacterial arabinan biosynthesis. Org Biomol Chem. 2007;5:2257-66.

32. Hsieh YSY, Zhang Q, Yap K, Shirley NJ, Lahnstein J, Nelson CJ, Burton RA Millar AH, Bulone V, Fincher GB. Genetics, Transcriptional Profiles, and Catalytic Properties of the UDP-Arabinose Mutase Family from Barley. Biochemistry. 2016;55:322-34

33. Rautengarten C, Ebert B, Herter T, Petzold CJ, Ishii T, Mukhopadhyay A, Usadel B, Scheller HV. The Interconversion of UDP-Arabinopyranose and UDP-Arabinofuranose Is Indispensable for Plant Development in Arabidopsis. Plant Cell. 2011;23:1373-90.

34. Castilho A, Pabst M, Leonard R, Veit C, Altmann F, Mach L, Glossl J, Strasser $\mathrm{R}$, Steinkellner H. Construction of a functional CMP-sialic acid biosynthesis pathway in Arabidopsis. Plant Physiol. 2008;147:331-9.

35. Roberts RM. The incorporation of D-glucosamine-14C into root tissues of higher plants. Plant Physiol. 1970;45:263-7.

36. Wold JK, Hillestad A. Demonstration of Galactosamine in a Higher Plant Cannabis-Sativa. Phytochemistry. 1976;15:325-26

37. Hori H. Demonstration of Galactosamine in Extracellular Hydroxyproline-Rich Macromolecule Purified from Culture Media of Tobacco Cells. Plant Cell Physiol. 1978:19:501-05.
38. Lenucci M, Leucci MR, Andreoli C, Dalessandro G, Piro G. Biosynthesis and characterization of glycoproteins in Koliella antarctica (Klebsormidiales, Chlorophyta). Eur J Phycol. 2006;41:213-22.

39. Yang Z, Bennett EP, Jorgensen B, Drew DP, Arigi E, Mandel U, Ulvskov $P$, Levery SB, Clausen H, Petersen BL. Toward Stable Genetic Engineering of Human O-Glycosylation in Plants. Plant Physiol. 2012;160:450-63.

40. Yang T, Echols M, Martin A, Bar-Peled M. Identification and characterization of a strict and a promiscuous $\mathrm{N}$-acetylglucosamine-1-P uridylyltransferase in Arabidopsis. Biochem J. 2010;430:275-84.

41. Zhang Q, Hrmova M, Shirley NJ, Lahnstein J, Fincher GB. Gene expression patterns and catalytic properties of UDP-D-glucose 4-epimerases from barley (Hordeum vulgare L.). Biochem J. 2006;394:115-24.

42. Agard NJ, Prescher JA, Bertozzi CR. A strain-promoted [3+2] azide-alkyne cycloaddition for covalent modification of blomolecules in living systems. J Am Chem Soc. 2004:126:15046-47.

43. Dommerholt J, van Rooijen O, Borrmann A, Guerra CF, Bickelhaupt FM, van Delft FL. Highly accelerated inverse electron-demand cycloaddition of electrondeficient azides with aliphatic cyclooctynes. Nat Commun. 2014;5:5378

44. Debets MF, van Berkel SS, Schoffelen S, Rutjes FPJT, van Hest JCM, van Delft FL. Aza-dibenzocyclooctynes for fast and efficient enzyme PEGylation via copper-free $(3+2)$ cycloaddition. Chem Commun. 2010;46:97-9.

45. Yao JZ, Uttamapinant C, Poloukhtine A, Baskin JM, Codelli JA, Sletten EM, Bertozzi CR, Popik W, Ting AY. Fluorophore Targeting to Cellular Proteins via Enzyme-Mediated Azide Ligation and Strain-Promoted Cycloaddition. J Am Chem Soc. 2012;134:3720-28.

46. Devaraj NK, Weissleder R, Hilderbrand SA. Tetrazine-based cycloadditions: application to pretargeted live cell imaging. Bioconjugate Chem. 2008;19:2297-9.

47. Patterson DM, Jones KA, Prescher JA. Improved cyclopropene reporters for probing protein glycosylation. Mol BioSyst. 2014;10:1693-97.

48. Späte A-K, Bußkamp H, Niederwieser A, Schart VF, Marx A, Wittmann V. Rapid Labeling of Metabolically Engineered Cell-Surface Glycoconjugates with a Carbamate-Linked Cyclopropene Reporter. Bioconjugate Chem. 2014:25:147-54

49. Murashige T, Skoog F. A Revised Medium for Rapid Growth and Bio Assays with Tobacco Tissue Cultures. Physiol Plant. 1962;15:473-97.

\section{Submit your next manuscript to BioMed Central and we will help you at every step:}

- We accept pre-submission inquiries

- Our selector tool helps you to find the most relevant journal

- We provide round the clock customer support

- Convenient online submission

- Thorough peer review

- Inclusion in PubMed and all major indexing services

- Maximum visibility for your research

Submit your manuscript at www.biomedcentral.com/submit
) Biomed Central 